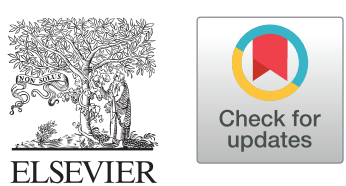

CJC Open 2 (2020) 189-191

\title{
Editorial
}

\section{Stroke Rehabilitation and Cardiac Rehabilitation: Siblings or Strangers?}

\author{
Jacqueline E. Stone, BPHE, BSc, MBA, MD, ${ }^{a}$ Sean Dukelow, BSc, MD, PhD, FRCPC, ${ }^{b}$ and \\ James A. Stone, BPHE, BA, MSc, MD, PhD, FRCPC ${ }^{b}$ \\ ${ }^{a}$ Cumming School of Medicine, University of Calgary, Calgary, Alberta, Canada \\ ${ }^{b}$ Libin Cardiovascular Institute of Alberta, Cumming School of Medicine, University of Calgary, Calgary, Alberta, Canada
}

See article by Toma et al., pages 195-206 of this issue.

Stroke rehabilitation (SR) and cardiac rehabilitation (CR) are either closely related relatives or strange bedfellows. Although the atherosclerotic origins of cardiovascular disease (CVD) produce similar degrees of morbidity and mortality in both of these populations, the significant motor and cognitive damages associated with large strokes, traditionally, have not seemed to lend themselves well to the cardiovascular conditioning and CVD risk factor management environments of CR. ${ }^{1}$ Yet, the devastating effects of stroke and heart attack would intuitively seem to lend themselves to a conjoint recovery, rehabilitation, and restoration environment.

The clear evidence supporting the use of thrombolytic therapy in acute strokes, followed more recently by the use of endovascular therapy, has brought the 2 acute-care worlds of myocardial infarction and stroke therapy closer together. ${ }^{2}$ An ever-increasing population of poststroke patients are being discharged from hospital with minimal physical and cognitive impairments. It is these emerging populations who may very well benefit from improvements in cardiorespiratory fitness, treatment target-driven control of CVD risk factors, along with mental health assessments and interventions where appropriate. $^{3}$

Over the last 3 decades, CR programs around the world have evolved from primarily exercise-only programs to comprehensive CVD management programs. As a direct consequence of this, these types of programs have clearly demonstrated their superiority over usual care in reducing mortality and rehospitalization. ${ }^{4}$ If similar programs of holistic chronic disease care could be developed and implemented for

Received for publication May 13, 2020. Accepted May 14, 2020.

Ethics Statement: To the best of our knowledge, the research reviewed or referenced within this article is adherent to the applicable and relevant ethical research guidelines.

Corresponding author: Dr James A. Stone, 4430 Macleod Trial SW, Calgary, Alberta T2G 0A4, Canada. Tel.: +1-587-430-0627; fax: +1-587430-0691.

E-mail: jastone@shaw.ca

See page 190 for disclosure information. patients who have suffered transient ischemic attacks or those who have only mild-to-moderate physical and cognitive impairments after stroke, it is entirely possible that these programs may similarly improve morbidity and mortality. A recent trial evaluating the impacts of a modified CR program in 783 stroke survivors demonstrated a markedly low 1-year poststroke mortality of $1.47 \%{ }^{5}$ compared with the US national rate of $31 \%,{ }^{6}$ possibly through improvements in both aerobic capacity and stroke-related function. Despite the purported benefits of CR, participation for stroke survivors remains low. Unfortunately, it is not as simple as: "If you build it they will come." Existing CR programs must be willing to see and treat patients with cerebral vascular disease, and practitioners must be willing to refer their patients to these clinical practice environments. Moreover, for those CR programs willing and capable of admitting cerebrovascular event patients to their programs, funding must follow the patients.

In this issue of CJC Open, Toma et al. ${ }^{7}$ report results from a large national survey of stroke patients' participation in CR programs. The authors aimed to characterize poststroke engagement in $\mathrm{CR}$, highlight individual program offerings, and identify barriers/facilitators to patient participation. The web-based survey was provided to $160 \mathrm{CR}$ programs across the country; $71 \%$ of contacted representatives responded, accounting for 130 programs in total. Of the respondent programs, most were in Ontario (39\%), in urban areas (44\%), and were hospital based/affiliated (60\%). Although response rates to the questionnaire were high, the number of stroke survivors attending CR programs was not. Only 65\% of CR programs accepted individuals with a diagnosis of stroke, and most $(62.5 \%)$ had fewer than 11 stroke survivors participate in the previous year. Only $25 \%$ of programs required patients to have a concurrent cardiac diagnosis, with the majority accepting a diagnosis of stroke alone. So how and why, in a country that sees approximately 50,000 new stroke cases a year, do only a few hundred patients attend CR programs?

Perhaps, unsurprisingly, the authors identify 2 critical barriers that seem to plague rehabilitation programs across the 
country: lack of both fiscal resources and staffing. But the story is more complex. The article by Toma et al. ${ }^{7}$ clearly identifies individuals excluded from most $\mathrm{CR}$ programs as those with severe mobility, communication, and/or cognitive deficits. Unfortunately, these happen to be individuals who, in many cases, may greatly benefit from $\mathrm{CR}$ programs. In some parts of the country, these individuals participate in community exercise programs run by not-for-profit organizations to increase and maintain cardiorespiratory fitness (eg, Together In Movement and Exercise). ${ }^{8}$ SR patients often transition to these programs after completing formal therapies.

Accommodating patients with more severe stroke-related impairments in $\mathrm{CR}$ programs necessitates staff training in poststroke communication, cognitive issues, and motor recovery, and may require the purchase of modified equipment. Toma et al. indicate that, to date, only a few CR programs in Canada have done so. This issue is particularly problematic for individuals who sustain concurrent stroke and myocardial infarction, or perioperative stroke during cardiac procedures, and are still unable to access CR programs. Although the authors identify who is and is not participating in existing CR programs, perhaps an important question to address is: which patients with stroke should definitely attend CR programs and how to best facilitate this?

However, the aforementioned restrictions regarding patient eligibility criteria do not fully explain low participation rates. Lack of referrals was another important barrier, and given that most programs do not have a limit on the number of stroke patients they can enrol, the authors argue for a better partnership between CR and SR. Over half of respondents indicated that their CR program was within $25 \mathrm{~km}$ of an SR facility, underscoring the ease of developing a more seamless partnership and referral process. Undoubtedly, there needs to be improved communication across medical specialties; specifically, physiatry, neurology, and cardiology, as well as all the other members of the multidisciplinary SR and CR teams.

Aerobic exercise in many SR programs has historically occurred in less than $25 \%$ of programs with programs typically focusing on facilitating neurologic recovery. ${ }^{8}$ Anecdotally, the authors have witnessed practice pattern changes over the last 5 years, where aerobic exercise seems to be increasingly integrated into SR. Updates to guidelines on incorporating aerobic exercise into SR have recently been released and provide guidance around exercise testing and monitoring. Interestingly, Toma et al. reveal that just under half of stroke patients in CR programs actually underwent a regular preparticipation aerobic assessment, which included a graded exercise test, with electrocardiogram and blood pressure monitoring, or 6-minute walk tests for persons with mobility deficits. In SR, our experience suggests that clinicians are often reluctant to prescribe exercise in stroke patients without baseline exercise testing and are uncomfortable performing this testing themselves. Alternatively, many SR programs lack access to adequate equipment and monitoring (ie, electrocardiogram and blood pressure monitoring) to perform these tests. In the face of limited resources, SR clinicians and rehabilitation team members should be educated in identifying patients who require pre-exercise testing as well as how to best facilitate/interpret these investigations. Once identified, these individuals could be referred to a CR program for assessment and then either retained within that program to undergo rehabilitation or transferred back to the SR program, depending on their particular clinically dominant rehabilitation requirements.

An important factor not highlighted by Toma et al. is that in SR, timing is critical. The brain undergoes a period of enhanced neural plasticity in the first few months after stroke. ${ }^{10}$ SR provided in the acute to subacute periods is thought to take advantage of this rewiring, such that stroke survivors can regain lost neurologic function. Intensity of therapy appears to play an important role in facilitating recovery. SR programs across the country take advantage of this by supplying the most intense therapy in the first few months after a stroke. Many of the stroke survivors discussed in the article by Toma et al. ${ }^{7}$ are well past the point (median 11 years after stroke) when we would not expect to see much in the way of neurologic recovery, unless they spent several hours daily performing hundreds of movements to develop new skills. ${ }^{11}$

Ultimately, the best timing for patients undergoing SR to consider CR needs further consideration. Importantly, CR programs have increasingly tried to shorten wait periods for patients, based on evidence regarding enhanced completion rates with reduced program delays, and multiple programs across the country now have effectively no waitlist. ${ }^{12}$ The wait time to begin CR in many programs in Canada is often dictated by patient delays rather than program delays. If, as suggested by Toma et al., toolkits and triage protocols could be developed that would facilitate rapid transition from acute care environments to ambulatory care environments, the number of home days for patients after acute stroke might be significantly improved through a blended care approach in SR and CR. Behind the scenes, this would require significant communication between SR and CR programs. From a patient perspective, however, the transitions of care in the rehabilitation program the patient is ultimately enrolled in, either SR or CR, should be seamless and clearly reflect the dominant needs of the patient.

Certainly, not everyone who has a stroke needs CR. Some stroke survivors are young, fit, and without cardiovascular risk factors. Toma et al. clearly highlight many of the challenges facing stroke survivors who would benefit from participation in CR programming. Although overlap exists between the components of SR and CR, clinical experience suggests that many patients would benefit from both. Identifying the best candidates, determining optimal timing for CR, and improving access are critical to optimizing patient recovery and secondary prevention. The goal should be to provide patients with the best possible outcome, which will require close collaboration across rehabilitative spheres.

\section{Funding Sources}

S.D. received funding from Heart and Stroke Foundation of Canada, Canadian Institutes of Health Research.

\section{Disclosures}

The authors have no conflicts of interest to disclose. 


\section{References}

1. Prior PL, Hachinski V, Unsworth K, et al. Comprehensive cardiac rehabilitation for secondary prevention after transient ischemic attack and mild stroke. Stroke 2011;42:3207-13.

2. Goyal M, Demchuk AM, Menon BK, et al. Randomized assessment of rapid endovascular treatment of ischemic stroke. N Engl J Med 2015;32: 1019-30.

3. Tang A, Marzolini S, Oh P, McIlroy WE, Brooks D. Feasibility and effects of adapted cardiac rehabilitation after stroke: a prospective trial. BMC Neurol 2010;10:40.

4. Martin BJ, Hauer T, Arena R, et al. Cardiac rehabilitation attendants and outcomes in coronary artery disease patients. Circulation 2012;126: 677-87.

5. Cuccurullo SJ, Fleming TK, Kostis WJ, et al. Impact of a stroke recovery program integrating modified cardiac rehabilitation on all-cause mortality, cardiovascular performance and functional performance. Am J Phys Med Rehabil 2019;98:953-63.

6. Fonarow GC, Smith EE, Reeves MJ, et al. Hospital-level variation in mortality and rehospitalization for Medicare beneficiaries with acute ischemic stroke. Stroke 2011;42:159-66.
7. Toma J, Hammond B, Chan V, et al. Inclusion of people poststroke in cardiac rehabilitation programs in Canada: a missed opportunity for referral. Can J Cardiol Open 2020;2:195-206.

8. Nathoo C, Buren S, El-Haddad R, et al. Aerobic training in Canadian stroke rehabilitation programs. J Neurol Phys Ther 2018;42:248-55.

9. Time Program Listings. Available at: https://www.uhn.ca/TorontoRehab/ Clinics/TIME/Documents/TR_PF_CC_TIME_Listings.pdf. Accessed May 10, 2020.

10. MacKay-Lyons M, Billinger SA, Enn JJ, et al. Aerobic exercise recommendations to optimize best practices in care after stroke: AEROBICS 2019 update. Phys Ther 2020;100:149-56.

11. Murphy TH, Corbett D. Plasticity during stroke recovery: from synapse to behaviour. Nat Rev Neurosci 2009;10:861-72.

12. Parker K, Stone JA, Arena R, et al. An early cardiac access clinic significantly improves cardiac rehabilitation participation and completion rates in low risk ST-elevation myocardial infarction patients. Can J Cardiol 2011;27:619-27. 